\title{
Bruxism and enuresis: common entities but understood
}

\section{Opinion}

In the population in general, especially in Pediatrics, there are a couple of sleep disorders that deserve different attention, due to the lack of medical culture to address them, these two conditions are bruxism and enuresis. The first one is very, very frequent to find it around us, either with any person that surrounds us, and it is very frequent in women, since it will increase the appearance of headaches in the population in general. This is defined as the involuntary habit of squeezing or grinding the teeth, especially during sleep; people in general, but even more serious, doctors or dentists have the misconception that it is quite parasite, and every time they come with this symptom to see take his "package- desparacites" ma s no question; and is that we speak of a phenomenon or condition that has a very definite origin in high levels of anxiety, which leads to a wear on the gums, temporomandibular joint dysfunction, headache or headaches that are very resistant to treatment and depression and anxiety for a chronic bad sleep. It is thought that only the famous " occlusal guard "prescribed in dental offices is the answer, but this is far from true, since it must be accompanied by exercise that allows for relaxation, and if they rush me, swimming is fantastic; supportive psychotherapy in many patients; sleep hygiene measures should be indicated, and always the review by a maxillofacial specialist; I always say this phrase to my patients who define the basis of their treatment "should be far from the person who is now and reinvent themselves" as this derives from their temperament and personality.

Enuresis is defined as the persistence of urination or urine without control beyond the age at which bladder control is achieved (in a range of 4 to 6 years old) and occurs both daytime way night, although the latter by far it is the most frequent, and it is cataloged within the group of parasomnias, which as we already mentioned are activation disorders or sudden sleep arousal. What causes enuresis? Although anatomical alterations in the urinary tract must be ruled out, mainly

\author{
Volume 2 Issue 3 - 2018
}

Gabriel Miranda Nava

Master in Public Health, Hospital Center of the Presidential General Staff, Mexico

Correspondence: Gabriel Miranda Nava, Military Doctor specialized in Neurology, Neurophysiology and sleeping diseases, Chief of Neurology of the Hospital Center of the Presidential General Staff, Mexico, Email drgabrielmiranda@hotmail.com

Received: May 21, 2018 | Published: June 07, 2018

and by far they are psychological, since it is the face of the depression in many children, that aside they present with anguish and feelings of guilt, believe me that it is quite painful for a child who lives This phenomenon, a situation that if we do not treat it with the maturity and stature required, will mark the life of this being for the rest of his life. Initially, the management of antidepressants may be indicated, but much more should be treated emotional aspects, with supportive psychotherapy, rewarding behavior of parents, which means not hitting or mortifying the child, and more than what is found, it is better to talk to him, explain that it is not his fault, and that he would receive some prize or encouragement if he could avoid it; Limit your fluid intake until certain time of day, exercise and our sleep hygiene measures.

\section{Acknowledgements}

None.

\section{Conflict of interest}

The author declares there is no conflict of interest. 